\title{
Optimal Channel Utilization Ratio in Ad Hoc Wireless Networks
}

\author{
G. Ferrari ${ }^{1,2}$ and O. K. Tonguz ${ }^{1}$ \\ ${ }^{1}$ Department of Electrical and Computer Engineering, Carnegie Mellon University, Pittsburgh, PA 15213-3890 USA \\ ${ }^{2}$ Department of Information Engineering, University of Parma, I-43100, Parma, Italy \\ E-mail: gianluigi.ferrari@unipr.it, tonguz@ece.cmu.edu \\ URL: www.tlc.unipr.it/ferrari, www.ece.cmu.edu/ tonguz/
}

\begin{abstract}
In this paper, we investigate the impact of the channel utilization ratio (CUR) on the performance of ad hoc wireless networks. Given that a node can hold a multi-hop route for a time interval defined as reserved channel utilization interval (RCUI), we assume that the node effectively utilizes the reserved route for an interval defined as effective channel utilization interval (ECUI), the duration of which corresponds to the duration of the message to be transmitted. This models a realistic scenario, where a node may use the shared radio medium for only a portion of the reservation interval. Defining the CUR as the ratio between the RCUI duration and the ECUI duration, we develop an analytical framework which leads to the evaluation of the "optimal" CUR for the maximization of the effective transport capacity, a concept recently introduced by the authors and representing the actual bandwidth-distance product carried by the network.
\end{abstract}

\section{INTRODUCTION}

Ad hoc wireless networks represent a new communication paradigm which has been developing over the last years. An important performance measure for this type of networks is the transport capacity, an information-theoretic concept introduced in [1], which can be viewed as the theoretical maximum bandwidth-distance product that a wireless network is capable of supporting. While most of the literature on ad hoc wireless networks has concentrated on medium access control (MAC) and routing protocols design [2], the physical layer plays a major role in influencing the performance of ad hoc wireless networks. In ad hoc wireless networks, a comprehensive crosslayer design is therefore needed to really capture the effect of the physical layer on higher layers. In [3], the author studies the impact of a Rayleigh fading channel on the design of routing protocols for ad hoc wireless networks. In [4]-[6], the impact of the physical layer is taken into account to design novel MAC protocols and to study the relationship between communication-theoretic quantities and network connectivity. In particular, the concept of effective transport capacity is introduced to quantify the "actual" bandwidth-distance product carried by the network [5].

In this paper, we investigate the impact of the channel utilization ratio (CUR) of the nodes (assuming a common behavior for all nodes) on the performance of ad hoc wireless networks. More precisely, we investigate the relation between the CUR and the effective transport capacity. After reserving a multi-hop route for its intended destination, a node is assigned a specific time interval to hold this route. This time interval is defined as reserved channel utilization interval (RCUI), during which the node has to transmit the desired message. The time effectively needed to transmit this information, i.e., the time duration of the message, is defined as effective channel utilization interval (ECUI). The ratio between the duration of the ECUI and the duration of the RCUI corresponds to the CUR. In this paper, we show that, given a particular network communication scenario, there exists an "optimal" CUR which maximizes the performance metric of interest, i.e., the effective transport capacity. The considered network communication model will be deliberately simple, in order to derive, in a relatively tractable analytical manner, meaningful insights into this interesting problem.

\section{BASIC ASSUMPTIONS}

- Before a node starts transmitting data to a desired destination, it must reserve a multi-hop route. After route reservation, ${ }^{1}$ a node can hold the route for an RCUI, the duration of which will be denoted in the following as $T_{\text {RCUI. }}$

- We assume that active multi-hop routes are disjoint. This simplifies the derivation of the effective transport capacity. However, the proposed approach can be extended to the case of crossing routes as well. In such a scenario, the queuing strategy at each node comes into play. Current ongoing research explores the extension of our framework in this direction [7].

- The maximum number of routes that can simultaneously be active will be denoted as $N_{\mathrm{R}}^{\max }$. In general, $\eta \leq N_{\mathrm{R}}^{\max }$ routes are active in the network.

- A relay node cannot transmit its own message while the source has not released the route.

- We assume that each node generates information at a constant bit-rate $\lambda_{b}$ (dimension: [b/s]). While this is somewhat unrealistic, it allows derivation of closed-form expressions which can also be applied, as guidelines, to scenarios where information is not generated at a constant rate.

- The transmission bit rate, denoted as $R_{b}$ (dimension: $[\mathrm{b} / \mathrm{s}])$, is the same for all nodes.

- For analytical purposes, perfect synchronization among the active nodes is considered. In other words, RCUIs of active source nodes are synchronous. Every $T_{\text {RCUI }}$, $\eta$ routes become active. The final result, in terms of

\footnotetext{
${ }^{1}$ Route reservation is assumed to be successfully accomplished, and our in this paper analysis focuses only on the transmission phase.
} 
existence of an optimal CUR, holds, however, also if this assumption is relaxed.

- Ideal fairness is assumed. More precisely, in a network communication scenario with $\eta$ active routes at a time, denoting by $N$ the total number of nodes in the network, the time between two successive transmissions of the same source node is $(N / \eta) T_{\mathrm{RCUI}}$. However, the obtained performance, in terms of optimal CUR, is the same regardless of the particular fairness policy enforced by the network.

- A message to be transmitted by a source node, after route reservation, is formed by a fixed number, denoted as $k$, of bits.

- The nodes are assumed to be static and placed at the vertices of a regular square grid: each node has four nearest neighbors (at the same distance). The extension to a scenario with random topology can be done following the approach presented in [8].

- Buffering is not explicitly considered. In other words, we are implicitly nodes with infinite buffers.

\section{Problem Statement}

Assume that a source node, after reserving a multi-hop route to its destination, holds it for a time interval of duration $T_{\text {RCUI }}$. The key question addressed in this paper is the following: given that the effective transmission lasts for an interval of duration $T_{\text {ECUI }}$ (where $T_{\text {ECUI }}<T_{\text {RCUI }}$ ), is there an "optimal" CUR (i.e., an optimal value of $T_{\text {ECUI }}$ ) such that the effective transport capacity is maximized? Considering the wireless network communication scenario characterized by the assumptions outlined in Section II, we will be able to answer this question.

A similar problem has been considered in [9], in the case of large and dense packet radio networks, and in [10] in the case of non-reservation-based radio packet networks based on an IEEE 802.11-type wireless network communication model. In order to reduce the collisions between ongoing communications, in [9] a common randomized transmission scheduling between communicating nodes is considered, whereas in [10] a pseudorandom time slot choice, based on the generation of a random seed exchanged between neighboring nodes, is used. We will show how our analytical results compare to those, based on the use of computer simulations, presented in [9], [10]. Insights into the relationship between CUR and network connectivity will also be provided, and it will be shown that they are in good agreement with recent results obtained with the use of percolation theory [11].

In order to analytically answer the key question of this paper, in the following section a discrete-time network communication model is presented [12].

\section{PRELIMINARIES}

\section{A. Topology and Routes}

We refer to the communication-theoretic framework developed in [4], [5]. We assume that a multi-hop route is constituted by a sequence of minimum-length hops (i.e., a node communicates directly only to one of its four neighboring nodes). Considering $N$ nodes over a circular area $A$, it is possible to show that the distance between two neighboring nodes, denoted as $r_{\text {link }}$, can be written (neglecting border effects) as $r_{\text {link }} \approx 1 / \sqrt{\rho_{S}}$, where $\rho_{S} \triangleq N / A$ is the node spatial density. In [4], it is also shown that each route is comprised, on average, of $\bar{n}_{\mathrm{h}}=\lfloor\sqrt{N / \pi}\rceil$ hops, where the notation $\lfloor\cdot\urcorner$ indicates the integer value closest to the argument. There can thus be, at most, $N_{\mathrm{R}}^{\max } \triangleq N / \bar{n}_{\mathrm{h}}=\lfloor\sqrt{N \pi}\rceil$ disjoint routes simultaneously active in the network. In general, there may be $\eta \leq N_{\mathrm{R}}^{\max }$ simultaneously active multi-hop routes.

\section{B. RCUI and ECUI}

The assumption of ideal fairness leads to a "perfectly cyclic" network communication behavior: there are $\eta$ disjoint routes simultaneously active for an interval of duration $T_{\mathrm{RCUI}}$; at the end of this interval, these routes are torn down and a new set of $\eta$ routes become active, and so on. Therefore, within an RCUI a node has to transmit the information (generated at a constant rate) accumulated in the previous $(N / \eta-1)$ RCUIs and the information which is being generated in the current RCUI. Hence, a node has to transmit the information which has been generated during $N / \eta$ intervals of duration $T_{\text {RCUI }}$. In the absence of perfect cyclicity among the nodes (i.e., non-ideal fairness), the analysis presented in the following would still hold, assuming that the size of a message to be transmitted by an active node was fixed-in this case, however, the assumption of constant-rate bit generation should be relaxed.

Assuming that the bit generation has constant rate $\lambda_{b}$, denoting as $k$ the amount of bits to be transmitted, it follows that $k=\lambda_{b} T_{\mathrm{RCUI}}(N / \eta)$. Considering a discrete-time model with basic time unit given by the bit duration $1 / R_{b}$, an RCUI can be associated with $n \triangleq R_{b} T_{\text {RCUI }}$ time units. The message with $k$ bits has to be transmitted within an RCUI, i.e., the condition $n \geq k$ has to be satisfied. This condition can be equivalently reformulated as

$$
\frac{R_{b}}{\lambda_{b}} \geq \frac{N}{\eta}
$$

If condition (1) is not satisfied, this implies that a node cannot transmit the entire message in an RCUI.

Based on condition (1), it follows that within the RCUI a node transmits (at a data-rate $R_{b}$ ) for an ECUI of duration

$$
T_{\mathrm{ECUI}} \triangleq \frac{k}{R_{b}}=\frac{\lambda_{b}}{R_{b}} \frac{N}{\eta} T_{\mathrm{RCUI}}
$$

from which one obtains the following expression for the CUR:

$$
\mathrm{CUR}=\frac{T_{\mathrm{ECUI}}}{T_{\mathrm{RCUI}}}=\frac{k}{n}=\frac{\lambda_{b}}{R_{b}} \frac{N}{\eta} .
$$

Based on (2), condition (1) can be equivalently rewritten as

$$
T_{\text {ECUI }} \leq T_{\text {RCUI }} \quad \text { or } \quad \text { CUR }<1 .
$$




\section{Effective Transport Capacity}

The effective transport capacity, introduced in [4], relates the network information flow to the network connectivity level. To this end, a useful indicator of network connectivity-the average sustainable number of hops-is introduced in [4], [5] and defined as follows:

$$
\bar{n}_{\mathrm{sh}} \triangleq \min \left\{\left\lfloor\frac{\ln \left(1-\mathrm{BER}^{\max }\right)}{\ln \left(1-\mathrm{BER}_{\text {link }}\right)}\right\rceil, \bar{n}_{\mathrm{h}}\right\}
$$

where $\mathrm{BER}^{\max }$ is the maximum acceptable BER at the end of a multi-hop communication route (this will be the physical layer-based quality of service, QoS, considered in this paper) and $\mathrm{BER}_{\text {link }}$ is the link BER. ${ }^{2}$ In other words, the average sustainable number of hops is the minimum between the maximum sustainable number of hops (which can be written as $\left.\left\lfloor\ln \left(1-\mathrm{BER}^{\max }\right) / \ln \left(1-\mathrm{BER}_{\text {link }}\right)\right\rceil\right)$ and the average number of hops $\bar{n}_{\mathrm{h}}$. Recalling that the hop length is $r_{\text {link }} \approx 1 / \sqrt{\rho_{S}}$, the average path length $\bar{r}_{\text {path }}$ can thus be written as

$$
\bar{r}_{\text {path }} \triangleq \bar{n}_{\text {sh }} r_{\text {link }} \approx \bar{n}_{\text {sh }} \sqrt{\frac{A}{N}} .
$$

At this point, we introduce the effective transport capacity, representing the actual bandwidth-distance product that can be sustained by the network [4], [5]. This quantity is obtained by combining the contributions from the various active routes, each one of which carries an "effective" data-rate equal to $\left(T_{\text {ECUI }} / T_{\text {RCUI }}\right) R_{b}=$ CUR $R_{b}$ over an average path length

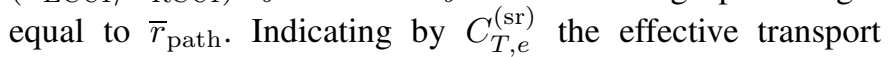
capacity associated with a single route and given that there are $\eta$ active routes, one can write

$$
C_{T, e} \triangleq \eta C_{T, e}^{(\mathrm{sr})}=\eta \mathrm{CUR} R_{b} \bar{r}_{\mathrm{path}} .
$$

The transport capacity, as defined in [1], represents the highest possible value of the effective transport capacity.

In order to evaluate the average sustainable number of hops $\bar{n}_{\text {sh }}$ (and, consequently, the effective transport capacity), the link BER has to be computed. This depends ultimately on the link SNR, which can be written as follows:

$$
\mathrm{SNR}_{\text {link }}=\frac{P_{\text {signal }}}{P_{\text {thermal }}+P_{\text {int }}}
$$

where: $P_{\text {signal }}$ is the received signal power at the ending node of a link and depends on the transmitted power $P_{t}$ at each node ${ }^{3}$ and on the propagation channel; $P_{\text {thermal }}$ is the thermal noise power at the receiver side; and $P_{\text {int }}$ is the internode interference (INI) power. We assume that the transmitted signal is affected by free space propagation loss and binary phase shift keying (BPSK) is the used modulation format (see [4], [5] for detailed expressions of $P_{\text {signal }}$ and $P_{\text {thermal }}$ in this case).

\footnotetext{
${ }^{2}$ We are implicitly assuming that the link BER is the same for all links. In a realistic communication scenario affected by interference, this is imprecise, since the BER depends on the position of the link in the network. An average interference analysis will be considered in the following, distinguishing between a best-case and a worst-case interference scenarios.

${ }^{3}$ Due to the flat architecture of an ad hoc wireless network, we assume that the transmission power is the same for all nodes.
}

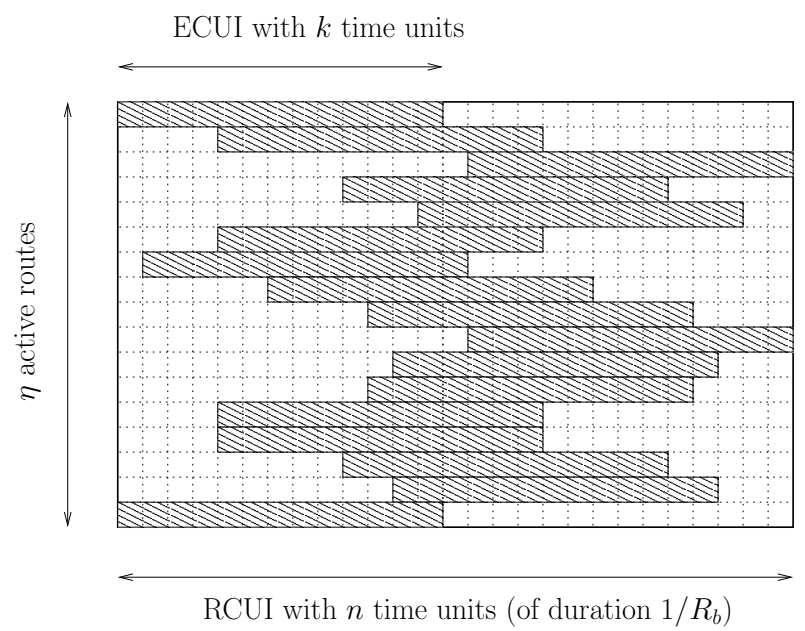

Fig. 1. $\quad \eta$ active routes transmitting in an RCUI of duration $T_{\text {RCUI }}$, with $n$ time units. Along each route a continuous message $k$ time units long is "flowing." In the figure, $\eta=17, n=27$, and $k=13$.

\section{Performance Analysis}

\section{A. Ideal Network Communication Scenario: No Interference}

An ideal scenario (where communications are not affected by INI), i.e., with $P_{\text {int }}=0$, would correspond to a scenario where the MAC protocol is "perfect," in the sense that a node accesses the shared radio medium without damaging any other active inter-node communication. In this case, the number $\eta$ of active routes has no influence on the interference: it only affects the effective transport capacity. It is easy to conclude that the highest effective transport capacity is obtained when a node transmits for the entire RCUI duration, i.e., when $T_{\text {ECUI }}=T_{\text {RCUI }}$ or, equivalently, when CUR $=1$. Intuitively, this is obvious: since there is no interference, a node can use the reserved route as much as possible. This will be confirmed by the results shown in Section VI.

\section{B. Realistic Network Communication Scenario: Interference}

In order to analyze this scenario, we assume that an active source node transmits its message, comprised of $k$ bits, continuously in the RCUI. This transmission strategy can be depicted through a matrix model, as shown in Fig. 1: each row corresponds to an active multi-hop route, while each column represents a time unit (i.e., a bit position) in the RCUI. Each filled position in this matrix model corresponds to a bit "flying" from source to destination in the route corresponding to the row. The model in Fig. 1 is based on the implicit assumption of neglecting the propagation delay and the processing time at each intermediate node of a multi-hop route. While neglecting the propagation delay is reasonable in wireless networks, neglecting the processing time at the intermediate nodes might not be so. If one wanted to take into account the processing time, the matrix-based model in Fig. 1 would still hold, provided that the time unit was larger than the bit duration $1 / R_{b}$ and took into account an average processing time at intermediate nodes. One could therefore extend straightforwardly the analysis proposed in the following. 
Assuming that each active source node starts transmitting independently from the other $\eta-1$ active source nodes in the network, and considering the possible positions of the first bit of each transmitted message, a "snapshot" of the network communication scenario, as depicted in Fig. 1, can be modeled as the outcome of an experiment where $\eta$ numbers between 1 and $(n-k+1)$, corresponding to the first bits of the $\eta$ messages, are extracted independently. Without loss of generality, one can restrict his/her attention to a specific multi-hop route, considering the probability of interference (from other routes) as a function of the bit position. We denote the probability of interference, from a single route, in the $i$ th bit position as $p_{\mathrm{I}}^{(i)}$. At this point, one has to evaluate the probability that a bit is transmitted by a source in the $i$-th position: we denote this probability ${ }^{4}$ as $p_{\mathrm{tx}}(i)$. After tedious but straightforward calculations, one can show that the average probability of bit interference can be written as

$\bar{p}_{\mathrm{I}} \triangleq \sum_{i=1}^{n} p_{\mathrm{tx}}(i) p_{\mathrm{I}}^{(i)}= \begin{cases}\frac{-n^{2}-4 k^{2}+5 n k-2 n+5}{3 k(n-k+1)} & k \leq n<2 k \\ \frac{-4 k^{2}+3 n k+3 k+1}{3(n-k+1)^{2}} & n \geq 2 k .\end{cases}$

It is easy to show that $\bar{p}_{\mathrm{I}}$ is an increasing function of the $\mathrm{CUR}=k / n$ and

$$
\lim _{\mathrm{CUR} \rightarrow 0} \bar{p}_{\mathrm{I}}=0 \quad \lim _{\mathrm{CUR} \rightarrow 1} \bar{p}_{\mathrm{I}}=1 .
$$

Moreover, for CUR $\ll 1$, it follows that $\bar{p}_{\text {I }} \simeq \mathrm{CUR}$.

The analysis conducted in the case of a single interfering route can be straightforwardly extended to the case of $m$ interfering routes (obviously, $m \in\{1, \ldots, \eta-1\}$ ). Denoting as $\overline{P r}_{\mathrm{I}}(m)$ the average probability of bit interference from $m$ routes, it is easy to conclude that the probability distribution $\left\{\overline{P r}_{\mathrm{I}}(m)\right\}_{m=1}^{\eta-1}$ is binomial with parameter $\bar{p}_{\mathrm{I}}$. Under the hypothesis that there are $m$ interfering active routes and that they are uniformly distributed over the network area, we simply assume (pessimistically) that the interference power is $m \bar{P}_{\text {int }}^{(\mathrm{sr})}$, where $\bar{P}_{\mathrm{int}}^{(\mathrm{sr})}$ is the interference power generated by a single interfering route (see [12] for more details). It is possible to show that the average per-route interference power can be bounded between a minimum and a maximum, corresponding to the best-case (such that only one node at a time is active in an interfering route) and the worst-case (such that all nodes of an interfering route are active at a time) interference scenarios, respectively. Both these minimum and maximum values do not depend on the CUR [12]. The average interference power $\bar{P}_{\text {int }}$ can therefore be expressed as

$$
\bar{P}_{\text {int }}=\sum_{m=1}^{\eta-1} m \bar{P}_{\text {int }}^{(\mathrm{sr})} \overline{\operatorname{Pr}}_{I}(m)=\bar{P}_{\text {int }}^{\text {(sr) }}(\eta-1) \bar{p}_{\mathrm{I}} .
$$

\section{Optimizing the Channel Utilization Ratio}

Based on expression (3) for the CUR, the aggregate effective transport capacity in (7) can be rewritten as

$$
C_{T, e}=\operatorname{CUR} \eta R_{b} \sqrt{\frac{A}{N}} \bar{n}_{\mathrm{sh}}
$$

${ }^{4}$ Note that the probability ensemble $\left\{p_{\mathrm{I}}^{(i)}\right\}_{i=1}^{n}$ is not a probability distribution as a function of $i$, whereas $\left\{p_{\mathrm{tx}}(i)\right\}_{i=1}^{n}$ is.
TABLE I

AD HOC WIRELESS NETWORK PARAMETERS ASSUMED.

\begin{tabular}{|l|c|}
\hline Antenna gains $\left(G_{t}, G_{r}\right)$ & 1 \\
\hline Carrier frequency $\left(f_{c}\right)$ & $2.4 \mathrm{GHz}$ \\
\hline Noise figure $(F)$ & $6 \mathrm{~dB}$ \\
\hline Transmitted power $\left(P_{t}\right)$ & $1 \mathrm{~mW}$ \\
\hline Network area $(A)$ & $1 \mathrm{~km}^{2}$ \\
\hline Number of nodes $(N)$ & 1000 \\
\hline Transmission data-rate $\left(R_{b}\right)$ & $1 \mathrm{Mb} / \mathrm{s}$ \\
\hline Maximum acceptable BER $\left(\mathrm{BER}^{\mathrm{max}}\right)$ & $10^{-3}$ \\
\hline
\end{tabular}

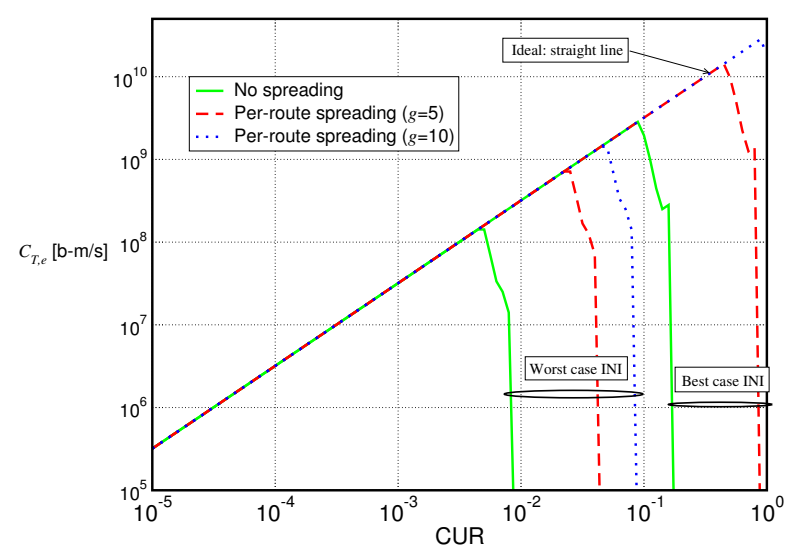

Fig. 2. Maximum effective transport capacity as a function of the CUR. A realistic case (with best-case and worst-case INI scenarios) and the ideal (no INI) case are considered. In a realistic scenario, the presence and absence of per-route spreading codes is considered.

Our analysis has shown that, even in a realistic network communication scenario (both in the worst-case and best-case interference scenarios), the highest effective transport capacity is obtained considering the maximum number (i.e., $\eta=N_{\mathrm{R}}^{\max }$ ) of active routes. Hence, in the following we limit ourselves to this case-the extension to a case with a different number of active routes is straightforward and the corresponding results will simply be a scaled version of those presented in the following. For $\eta=N_{\mathrm{R}}^{\max }=N / \bar{n}_{\mathrm{h}}$, the aggregate effective transport capacity in (11) can be equivalently rewritten as

$$
C_{T, e}=\operatorname{CUR} R_{b} \sqrt{\pi A} \bar{n}_{\mathrm{sh}} .
$$

We now evaluate the effective transport capacity as a function of the CUR: the "optimal" value of the CUR will correspond to the overall maximum of the effective transport capacity. The values assumed for the major network parameters are shown in Table I. In Fig. 2, the effective transport capacity is shown as a function of the CUR. The behavior of the effective transport capacity is considered in ideal (no INI) and realistic (with INI) cases. Note that this behavior does not depend on the specific message length. In fact, if the message length $k$ is fixed, varying the CUR corresponds to varying the RCUI duration; if, instead, the RCUI duration is fixed (i.e., $n$ is given), varying the CUR corresponds to varying the message length.

As expected, in the ideal case (i.e., when the interference power is equal to zero) the maximum effective transport capacity is proportional to the CUR (see (12)) and reaches its 
TABLE II

CRUCIAL VALUES OF THE CUR IN A REALISTIC SCENARIO.

\begin{tabular}{|l|c|c|}
\hline & $\begin{array}{c}\text { Worst-case } \\
\text { Interference }\end{array}$ & $\begin{array}{c}\text { Best-case } \\
\text { Interference }\end{array}$ \\
\hline \hline No per-route spreading & $0.48 \%$ & $8.8 \%$ \\
\hline Per-route spreading $(g=5)$ & $2.5 \%$ & $44 \%$ \\
\hline Per-route spreading $(g=10)$ & $4.9 \%$ & $88 \%$ \\
\hline
\end{tabular}

maximum when $\mathrm{CUR}=1$. For the realistic case, two possible communication strategies are considered.

1. Each multi-hop route is assigned a spreading code [13]. As an example, we consider two possible values for the spreading factor, namely $g=5$ and $g=10$, and we simply assume that the interference power is reduced by a factor equal to $g$-this is valid, provided that the used spreading codes are distributed uniformly among the routes (which is reasonable, assuming that the number of routes is sufficiently large and each route selects a spreading code randomly ${ }^{5}$ ). Obviously, the use of per-route spreading codes requires that the available bandwidth $B$ is sufficiently large ( $\left.B \simeq g R_{b}\right)$.

2. No per-route spreading code is used: all routes interfere "completely" with each other.

In Fig. 2, for both of these communication strategies, lower and upper bounds, corresponding to worst-case and best-case interference scenarios, respectively, are shown. It is interesting to observe that, in all realistic cases, the "shape" of the curve is the same: it is proportional to the CUR up to a point, corresponding to a critical value of the CUR, beyond which the maximum effective transport capacity rapidly decreases, due to the increase of interference. The "optimal" value of CUR is the value corresponding to the maximum of the curve.

The optimal CUR values in the realistic cases shown in Fig. 2 are summarized in Table II, distinguishing between worst-case and-best case interference scenarios. Note that in [9], [10] the CUR for optimized network performance is around $30 \%$. Considering the results in Table II, and assuming that the actual networking behavior is somewhere in the middle between worst-case and best-case scenarios, it is possible to conclude that the optimal CUR in the proposed network communication scenario with disjoint routes and per-route spreading codes is around $23 \%$ if $g=5$ and about $46 \%$ if $g=10$. Therefore, considering a spreading factor between 5 and 10, one expects the average performance of our ad hoc wireless network communication scheme to be similar to that of the schemes considered in [9], [10]. While the schemes in [9], [10] use scheduling mechanisms to reduce the interference, our scheme, instead, makes use of per-route spreading codes to achieve that. The final results, however, are in general agreement.

\section{Discussion}

\section{A. Interference/Thermal Noise Tradeoff}

In a realistic scenario, the behavior of each of the effective transport capacity curves in Fig. 2 has a typical trend: it

\footnotetext{
${ }^{5}$ In an IS-95 cellular system, the spreading factor is usually much higher than 10 [13].
}

grows linearly, and then it rapidly falls down to zero. In other words, for either very little or very large values of the CUR, the effective transport capacity is very small. In order to understand this behavior, we first observe the following facts. For a given network area $A$, from (12) one can write:

$$
C_{T, e} \propto \operatorname{CUR} R_{b} \bar{n}_{\mathrm{sh}}
$$

in which $\bar{n}_{\mathrm{sh}}$ is a function of the link SNR in (8), where $P_{\text {thermal }} \propto R_{b}$ and $P_{\text {int }}$ is an increasing function of the CUR (almost linear for low values of the CUR). At this point, one can understand what happens for varying values of the CUR.

- For low values of the CUR, the interference power is negligible, and the total noise power is given by the thermal noise power. In particular, $C_{T, e}$ grows almost linearly with the CUR. This means that $R_{b}$ is almost constant and $P_{\text {thermal }}$ is sufficiently low to guarantee full connectivity, i.e., $\bar{n}_{\mathrm{sh}}=\bar{n}_{\mathrm{h}}=18$.

- The optimal value of the CUR corresponds to a scenario where thermal noise and interference powers are, together, still sufficiently low to guarantee full connectivity. However, for values of CUR larger than the optimal, the interference power becomes too large, and connectivity is lost. This is shown in Fig. 3, where the average number of sustainable hops $\bar{n}_{\mathrm{sh}}$ is shown. In the ideal case, the average number of sustainable hops is equal to the average number $\bar{n}_{\mathrm{h}}$ for all CUR values. Loss of connectivity, i.e., sudden drop of $\bar{n}_{\mathrm{sh}}$, leads to rapid deterioration of the effective transport capacity to zero. This sudden loss of connectivity, for increasing CUR, i.e., for increasing interference, is in agreement with the conclusions, based on percolation theory, presented in [11].

Summarizing: for low values of CUR, the shared resource (radio channel) is underutilized, there is full connectivity and the effective transport capacity is low; for large values of CUR, the channel is overutilized, the interference level is too large, connectivity is lost and the effective transport capacity drops to zero. There is a sharp transition between these two regions, and the optimal CUR is exactly in the middle.

\section{B. Impact of Spreading}

Careful inspection of the results shown in Fig. 2 reveals that the improvement brought by using per-route spreading codes with $g=10$ is not twice as large as that brought by using per-route spreading codes with $g=5$. In other words, this means that it is sufficient to consider a relatively limited set of per-route spreading codes to significantly improve the network performance. Use of larger and larger sets of perroute spreading codes leads, in relative terms, to smaller and smaller performance improvement.

\section{Impact of Transmit Power}

The results shown in Fig. 2 and Fig. 3 are obtained considering a transmit power equal to $P_{t}=1 \mathrm{~mW}$ (common for all nodes). This corresponds, for the considered node spatial density $\left(\rho_{S}=10^{-3} \mathrm{~m}^{-2}\right)$, to requiring that the receiver 


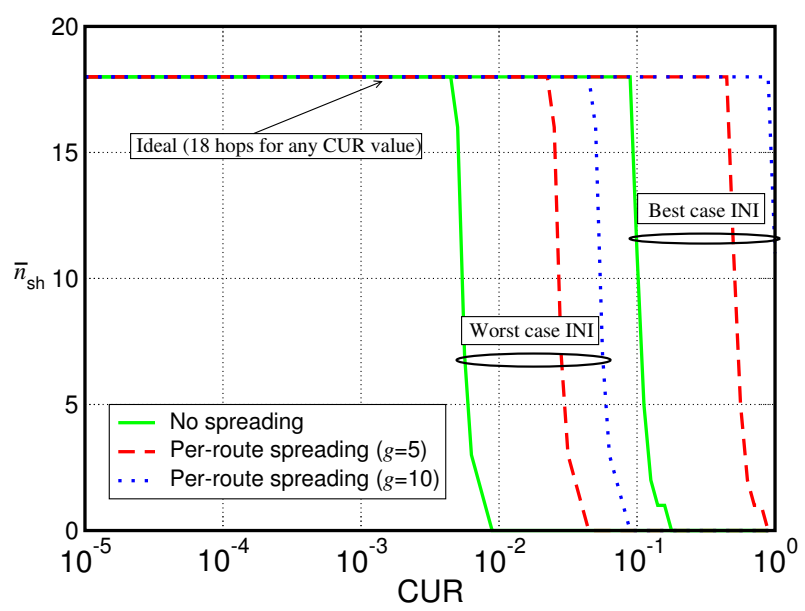

Fig. 3. Average sustainable number of hops as a function of the channel utilization ratio. A realistic case (with best-case and worst-case INI scenarios) and the ideal (no INI) case are considered.

sensitivity of a node is around $-110 \mathrm{dBm}$. Our results on the impact of the transmit power on the network performance (not shown here for lack of space) lead to the following conclusions.

- Increasing the transmit power (for example, considering $P_{t}=10 \mathrm{~mW}$ and a receiver sensitivity of $-100 \mathrm{dBm}$ ) leads to negligible performance improvement, in terms of effective transport capacity. In fact, the only effect is that of making loss of connectivity faster for values of CUR higher than the optimal value. This is due to the fact that in the region where interference power dominates and connectivity is lost, i.e., for values of CUR larger than the optimal, the link SNR becomes insensitive to the transmit power (since both $P_{\text {signal }}$ and $P_{\text {int }}$ are proportional to $P_{t}$, and $P_{\text {thermal }}$ is negligible). In other words, this means that increasing the transmit power corresponds to a waste of resources, especially in terms of battery consumption at the nodes.

- On the other hand, if the transmit power is reduced, it turns out that the critical behavior around the optimal CUR value (i.e., full connectivity below it and loss of connectivity right above it) disappears. More precisely, the optimal CUR value is no longer a critical connectivity value; instead, there is also a region, above this optimal value, where connectivity is still preserved. In other words, connectivity does not break suddenly as soon as the CUR slightly overcomes the optimal CUR value. In this reduced transmit power scenario, the optimal CUR corresponds to a stable network behavior: small oscillations of the actual CUR around the optimal value do not lead to loss of connectivity. Reducing the transmit power, however, requires a proportional increase of the receiver sensitivity, and this might be unrealistic for a practical ad hoc wireless network. In order to keep the same receiver sensitivity, one possibility would be the use of directional antennas. This research direction is currently under investigation.

\section{CONCLUDING REMARKS}

The impact of the CUR on the performance of ad hoc wireless networks has been investigated, considering an ideal scenario (without INI) and a realistic scenario (with INI). Our results have shown the existence of an optimal intermediate value of the CUR for the maximization of the effective transport capacity, such that radio resource underutilization and excessive interference are best balanced. Our results show that use of per-route spreading codes with relatively low spreading factor might lead to significant performance improvement. On the other hand, it is important to understand that increasing the transmit power beyond a critical point does not increase the effective transport capacity.

As mentioned in the introduction, the impact of the CUR on the network performance has been analyzed via several simplifying assumptions. However, even relaxing these assumptions, we expect that it would still be possible to identify an "optimal" CUR in the corresponding wireless network communication scenario. Our current research is focused on exploring this interesting extension.

\section{REFERENCES}

[1] P. Gupta and P. R. Kumar, "The capacity of wireless networks," IEEE Trans. Inform. Theory, vol. 46, pp. 388-404, March 2000.

[2] C. E. Perkins, Ad hoc Networking. Upper Saddle River, NJ, USA: Addison-Wesley, 2001.

[3] M. Haenggi, "The impact of power amplifier characteristics on routing in random wireless networks," in Proc. IEEE Global Telecommun. Conf. (GLOBECOM), San Francisco, CA, USA, December 2003, pp. 513517.

[4] G. Ferrari and O. K. Tonguz, "Performance of circuit-switched ad hoc wireless networks with Aloha and PR-CSMA MAC protocols," in Proc. IEEE Global Telecommun. Conf. (GLOBECOM), San Francisco, USA, December 2003, pp. 2824 - 2829.

[5] — , "MAC protocols and transport capacity in ad hoc wireless networks: Aloha versus PR-CSMA," in Proc. IEEE Military Comm. Conf. (MILCOM), vol. 2, Boston, USA, October 2003, pp. 1311 - 1318.

[6] O. K. Tonguz and G. Ferrari, Ad Hoc Wireless Networks: A Communication-Theoretic Perspective. John Wiley \& Sons, 2005, in press.

[7] S. Panichpapiboon, G. Ferrari, N. Wisitpongphan, and O. K. Tonguz, "Route reservation in ad hoc networks: Is it a good idea?" in Proc. IEEE Wireless Commmunications and Networking Conference (WCNC), New Orleans, LA, USA, March 2005, to appear.

[8] S. Panichpapiboon, G. Ferrari, and O. K. Tonguz, "Impact of interference in uniform and random ad hoc networks," in Proc. IEEE Vehicular Tech. Conf. (VTC), Milan, Italy, April 2004, also available at www. ece.cmu.edu/ tonguz/.

[9] T. J. Shepard, "A channel access scheme for large dense packet radio networks," in Proc. ACM Conference of the Special Interest Group on Data Communication (SIGCOMM), Palo Alto, CA, 1996, pp. 219-230.

[10] R. Rozovsky and P. R. Kumar, "SEEDEX: a MAC protocol for ad hoc networks," in Proc. ACM Int. Symp. on Mobile Ad Hoc Network. and Comput. (MOBIHOC), Long Beach, CA, USA, 2001, pp. $67-75$.

[11] O. Dousse, F. Baccelli, and P. Thiran, "Impact of interferences on connectivity in ad hoc networks," in Proc. IEEE Conf. on Computer Commun. (INFOCOM), San Francisco, USA, April 2003, pp. 1724 1733.

[12] G. Ferrari and O. K. Tonguz, "Circuit-switched sensor networks: A combinatorial analysis for randomized transmission," in Proc. IEEE International Conf. on Commun. (ICC), vol. 7, Paris, France, June 2004, pp. $4357-4361$.

[13] N. Abramson, "Multiple access in wireless digital networks," Proc. IEEE, vol. 82, pp. 1360-1370, September 1994. 